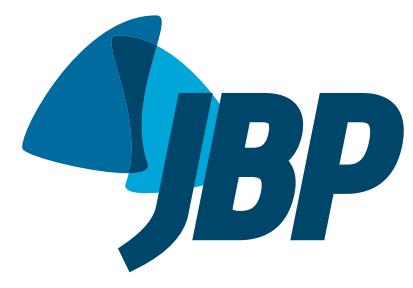

\title{
Clinical, epidemiological, and etiological profile of inpatients with community- acquired pneumonia in a public hospital in the interior of Brazil
}

\author{
Laura Fuchs Bahlis 1,2,3,a, Luciano Passamani Diogo ${ }^{3, b}$, \\ Ricardo de Souza Kuchenbecker ${ }^{4, c}$, Sandra Costa Fuchs ${ }^{4, d}$
}

1. Faculdade de Medicina, Universidade do Vale do Rio dos Sinos, Campus São Leopoldo, São Leopoldo (RS) Brasil.

2. Programa de Pós-Graduação em Epidemiologia, Universidade Federal do Rio Grande do Sul - UFRGS Porto Alegre (RS) Brasil.

3. Hospital de Clínicas de Porto Alegre, Porto Alegre (RS) Brasil.

4. Faculdade de Medicina, Universidade Federal do Rio Grande do Sul - UFRGS - Porto Alegre (RS) Brasil.

a. (D) http://orcid.org/0000-0002-1986-0970

b. (ID) http://orcid.org/0000-0001-6304-2767

c. (iD http://orcid.org/0000-0002-4707-3683

d. (D) http://orcid.org/0000-0001-6351-9588

Submitted: 15 December 2017 Accepted: 18 April 2018

Study carried out under the auspices of the Programa de Pós-Graduação em Epidemiologia, Universidade Federal do Rio Grande do Sul - UFRGS -

Porto Alegre (RS) and at the Hospital Montenegro, Montenegro (RS) Brasil.

\begin{abstract}
Objective: To describe the patient profile, mortality rates, the accuracy of prognostic scores, and mortality-associated factors in patients with community-acquired pneumonia (CAP) in a general hospital in Brazil. Methods: This was a cohort study involving patients with a clinical and laboratory diagnosis of CAP and requiring admission to a public hospital in the interior of Brazil between March 2014 and April 2015. We performed multivariate analysis using a Poisson regression model with robust variance to identify factors associated with in-hospital mortality. Results: We included 304 patients. Approximately $70 \%$ of the patients were classified as severely ill on the basis of the severity criteria used. The mortality rate was $15.5 \%$, and the ICU admission rate was $29.3 \%$. After multivariate analysis, the factors associated with in-hospital mortality were need for mechanical ventilation (OR: 3.60; 95\% Cl: 1.85-7.47); a Charlson Comorbidity Index score > 3 (OR: 1.30; 95\% Cl: 1.18-1.43); and a mental Confusion, Urea, Respiratory rate, Blood pressure, and age > 65 years (CURB-65) score $>2$ (OR: 1.46; 95\% CI: 1.09 1.98). The mean time from patient arrival at the emergency room to initiation of antibiotic therapy was $10 \mathrm{~h}$. Conclusions: The in-hospital mortality rate of $15.5 \%$ and the need for ICU admission in almost one third of the patients reflect the major impact of CAP on patients and the health care system. Individuals with a high burden of comorbidities, a high CURB-65 score, and a need for mechanical ventilation had a worse prognosis. Measures to reduce the time to initiation of antibiotic therapy may result in better outcomes in this group of patients.
\end{abstract}

Keywords: Community-acquired infections; Pneumonia; Hospital mortality; Risk factors.

\section{INTRODUCTION}

Community-acquired pneumonia (CAP) is one of the leading causes of death from infectious diseases worldwide. ${ }^{(1)}$ It is a public health problem and is a cause of morbidity and mortality in all age groups. ${ }^{(2)}$ Mortality rates vary according to the population and context studied, ranging from $1 \%$, in outpatients, to $50 \%$, in inpatients. ${ }^{(3,4)}$ High mortality rates are more common in developing countries, such as Brazil, Argentina, and India. ${ }^{(5,6)}$ CAP is responsible for high costs in public and private health care facilities, whether in outpatient clinics, emergency rooms, or hospital wards. ${ }^{(7,8)}$ In Brazil, data from the Brazilian Unified Health Care System show that pneumonia was the second leading cause of hospitalization in 2017, accounting for approximately $14 \%$ of all hospitalizations. ${ }^{(9)}$

Although CAP is a high-incidence disease, with multiple studies having been conducted on risk factors and available therapies, several issues regarding CAP remain controversial.(10) Using guidelines for the treatment of pneumonia has been shown to reduce hospital stays, mortality rates, and complications rates. ${ }^{(11-13)}$ However, a recent study conducted in Brazil showed that slightly more than half of the patients admitted to a university hospital were treated in accordance with current guidelines. ${ }^{(14)}$ With regard to assessment of disease severity, studies have shown that using clinical judgment alone can either underestimate or overestimate the severity of the clinical presentation. This strategy can lead to unnecessary hospitalizations, as well as to interventions that are less aggressive than those required in more severe cases, culminating in negative outcomes. ${ }^{(15-17)}$ Nevertheless, a study published in 2015 showed that most physicians in Brazil use clinical judgment alone to assess disease severity in patients with CAP. ${ }^{(14)}$

It is possible to suspect that the aforementioned discrepancies are partly due to the fact that the major guidelines and severity scores are based on large international studies, and, despite the high reported incidence of CAP in Brazil, little is known about local microbiological patterns and disease severity at the regional level. ${ }^{(18)}$ Therefore, we conducted a cohort study involving patients with CAP admitted to a hospital in the interior of Brazil over a one-year period, in order to describe the patient profile, mortality rates, the accuracy 
of prognostic scores, and factors associated with in-hospital mortality in these patients.

\section{METHODS}

\section{Study population}

The present study was conducted in a secondary-care general hospital, with 130 beds, located in the city of Montenegro, Brazil. The hospital provides care via the Brazilian Unified Health Care System to approximately 160,000 people, many of whom reside in one of 19 surrounding municipalities. In 2015, respiratory infections represented the leading cause of admission to the hospital, and the rate of in-hospital mortality was $10.2 \%{ }^{(19)}$

\section{Study design}

We screened for patients who were 14 years of age or older, had respiratory symptoms, and were referred for hospital admission between May 2014 and April 2015. Patient inclusion in the cohort at baseline was based on a clinical and radiographic diagnosis of CAP and a referral by the attending physician for hospitalization. We excluded patients with nosocomial pneumonia-characterized by hospitalization for 2 or more days in the past three months-residents of nursing or retirement homes; those who received intravenous antibiotics, chemotherapy, or scar treatment in the past 30 days; and those undergoing renal replacement therapy. Patients were assessed using the following severity scores: Charlson Comorbidity Index (CCI); mental Confusion, Urea, Respiratory rate, Blood pressure, and age > 65 years (CURB-65) score; and Pneumonia Severity Index (PSI), on the basis of data documented in the cohort's medical records collected at baseline. We assessed the clinical course of patients during in-hospital follow-up and determined clinical outcomes at the time of hospital discharge.

Cases of pneumonia were defined in accordance with the criteria established by the Centers for Disease Control and Prevention, ${ }^{(20)}$ on the basis of chest X-rays with at least one of the following findings: new or progressive and persistent infiltrate, consolidation, and/or cavitation; and at least one of the following signs or symptoms: fever $\left(>38^{\circ} \mathrm{C}\right)$ with no other cause detected; leukopenia $\left(<4,000\right.$ leukocytes $\left./ \mathrm{mm}^{3}\right)$ or leukocytosis ( $\geq 12,000$ leukocytes $\left./ \mathrm{mm}^{3}\right)$; and, for adults aged 70 years or older, a change in mental state with no other cause identified. In addition, there should be at least two of the following findings: new onset of purulent sputum or change in character of sputum; increased respiratory secretions; increased frequency of aspiration; onset or worsening of cough, dyspnea, or tachypnea; bronchial breath sounds; worsening of gas exchange (e.g., oxygen desaturation, with a $\mathrm{PaO}_{2} /$ $\mathrm{FiO}_{2}$ ratio $\leq 240$ ); increased need for oxygen; or need for mechanical ventilation.

The study was approved by the Porto Alegre Hospital de Clínicas Research Ethics Committee (Protocol GPPG no. 150168), which is accredited by the Office of Human Research Protections as an institutional review board, and researchers signed a data use agreement protecting the confidentiality of medical records.

\section{Study variables}

Clinical, laboratory, and radiological data for the first $24 \mathrm{~h}$ following the emergency room visit were obtained from a review of medical records, and included the following variables: age; gender; place of residence; $\mathrm{RR}$; arterial blood pressure; axillary temperature; $\mathrm{HR}_{\text {; }}$ presence of mental confusion; $\mathrm{SpO}_{2}$; comorbidities (as documented by the attending physician); history of hospitalization; chest X-ray findings (as assessed by a radiologist); and laboratory tests ordered in the emergency room. Laboratory tests included arterial blood gas analysis, urea, serum creatinine, blood glucose, sodium, and blood count. We recorded the antibiotics administered during hospitalization, as well as total length of hospital stay, length of ICU stay, and need for mechanical ventilation. The primary outcome was all-cause in-hospital mortality, as documented in the medical records and confirmed by review of hospital discharge summaries or death certificates, as appropriate. All patients had medical records, and their discharge summaries were completed by the attending physician within $48 \mathrm{~h}$ of discharge.

It is important to emphasize that patient management, treatment choice, and outcomes suffered no interference from this study, because it was an observational study and we had no contact with either the patients or the attending physicians, who were responsible for all clinical decisions. In the hospital, there is a care protocol for the treatment of pneumonia, and inpatients are treated by a physician team, which was composed of five members at the time of the study.

\section{Data analysis}

Data were entered into an Excel ${ }^{\circledR}$ database by two different individuals and subsequently compared to identify possible typographical errors. Continuous variables are expressed as mean and standard deviation; categorical variables are expressed as frequency and proportion. The bivariate analysis between clinical characteristics and mortality was performed by using the Student's t-test (for means and standard deviations) or Pearson's chi-square test (for proportions). ROC curve analysis was performed to evaluate the prognostic indices. A Poisson regression model with robust variance was used to assess the relationship between the variables and the primary outcome (in-hospital mortality). Analyses with a two-tailed $p$ value $<0.05$ were considered statistically significant.

All analyses were performed with the Statistical Package for the Social Sciences, version 17.0 (SPSS Inc., Chicago, IL, USA) and the R software, version 4.0-1 (The R Foundation for Statistical Computing, Vienna, Austria). ${ }^{(21-24)}$

\section{RESULTS}

Between March 2014 and April 2015, we assessed 459 patients with respiratory infection, 155 of whom did 
not meet the diagnostic criteria for CAP, and, therefore, 304 patients were included in the final analysis (Figure $1)$. Of those, 171 (56\%) were male, and the mean age was $67.1 \pm 17.2$ years. Most participants (69\%) resided in the city of Montenegro. Of the patients in the final sample, 150 (49\%) had asthma or COPD (previous lung disease), and 155 (51\%) were smokers. The mean CCI score was $4.9 \pm 3.1$. The characteristics of the patients are presented in Table 1.

The mean CURB-65 score was $2.2 \pm 1.1$, and $71 \%$ of the patients were considered to have severe CAP (CURB-65 scores $\geq 2$ ). The mean PSI score was 3.8 \pm 1.3 , and $74 \%$ of the patients were considered to have severe CAP (PSI scores $>3$ ). The two indices showed good ability to predict in-hospital mortality, with areas under the ROC curve being 0.73 for the CURB-65 score (95\% CI: 0.66-0.80; $p<0.001)$ and 0.75 for the PSI ( $95 \%$ CI: $0.68-0.82 ; p<0.001)$; there was no statistically significant difference between the indices $(p=0.65)$, as shown in Figure 2 .

The mean time from patient arrival at the emergency room to initiation of antibiotic therapy was $10.4 \pm 7.7$ h. Blood or sputum samples were collected for culture from 101 patients (33\%), and the infectious agent was isolated in 53 patients (17\%). The most commonly isolated agent was Streptococcus pneumoniae (in $36 \%$ of positive cultures). The most commonly used antibiotic regimen was amoxicillin plus clavulanate,

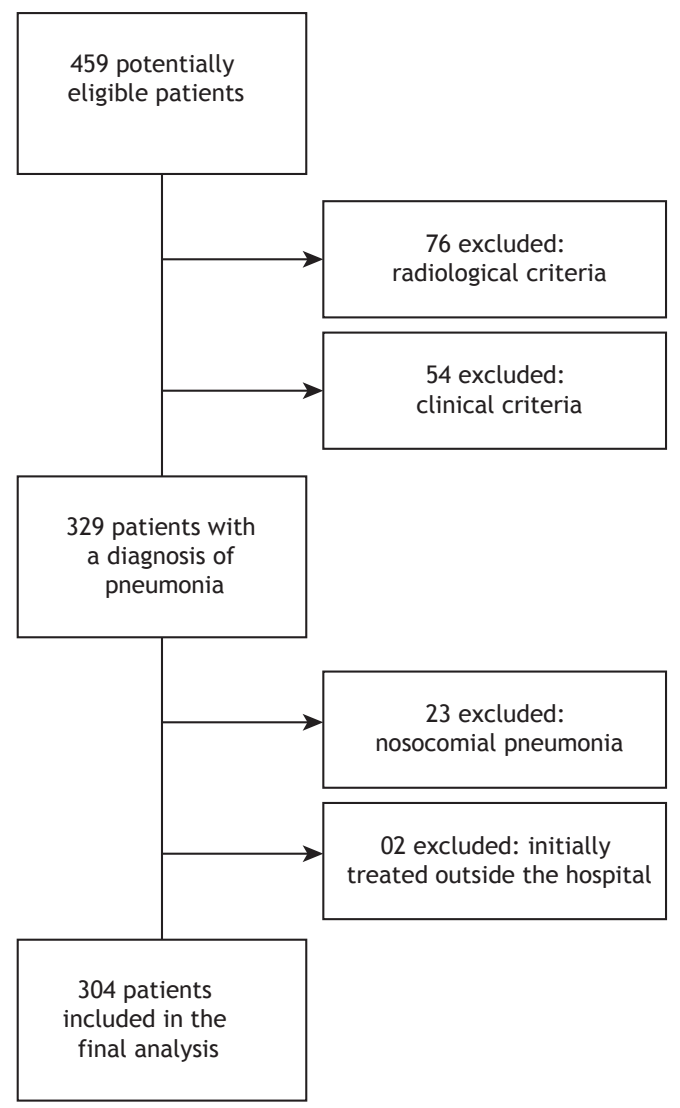

Figure 1. Flow chart of patient inclusion in the study. in 219 patients (72\%), followed by azithromycin, in $200(66 \%)$.

We found that 191 (63\%) of the patients required a change in the antibiotic regimen during hospitalization. The most common change was the addition of azithromycin to the antimicrobial regimen, on the basis of clinical assessment by the attending physician. During the in-hospital follow-up period, 47 (15.5\%) of the patients died, $89(29.3 \%)$ required ICU treatment, and 98 (32.2\%) underwent mechanical ventilation. The mean length of hospital stay was $7.2 \pm 7.3$ days (median, 5 days).

Univariate analysis showed that the factors associated with increased risk for in-hospital mortality were: a CCI score > 3 (OR: 7.18; 95\% CI: 2.28-22.58; p < 0.001 ); a CURB-65 score > 2 (OR: 4.45; 95\% CI: 1.64-12.02; $p=0.001$ ); a PSI score > 3 (OR: 9.05; 95\% CI: $1.27-64.14 ; p=0.004)$; need for a change in the antibiotic regimen (OR: $2.15 ; 95 \% \mathrm{CI}: 1.05-4.42$; $\mathrm{p}=0.02$ ); need for mechanical ventilation (OR: 6.13 ; 95\% CI: 3.33-11.28; $\mathrm{p}<0.001)$; age > 62 years (OR: 6.73; 95\% CI: 2.35-19.34; $\mathrm{p}<0.001$ ); and being institutionalized (OR: $2.82 ; 95 \% \mathrm{CI}: 1.08-7.35$; $p=0.03$ ).

After multivariate analysis, the factors that remained associated with in-hospital mortality were need for mechanical ventilation (OR: 3.58; 95\% CI: 1.92-6.67; p < 0.001); a CCI score > 3 (OR: 1.30; 95\% CI: 1.22-1.39; $\mathrm{p}<0.001)$; and a CURB-65 score > 2 (OR: $1.45 ; 95 \%$ CI: $1.05-2.00 ; p=0.04$; Table 2 ).

\section{DISCUSSION}

CAP continues to be one of the leading causes of death from infectious disease worldwide. Despite the large number of international studies on this subject, there are few studies describing the impact of CAP on patients and the characteristics of the disease in Brazil, especially in inpatient units. In our study, we assessed inpatients with CAP in a secondary-care hospital in Brazil over a one-year period. The observed in-hospital mortality rate of $15.5 \%$ reflects the major impact of this disease, being similar to that found in other national and international studies. ${ }^{(25,26)}$ In addition, ICU admission was required in almost one third of the cases $(29.3 \%)$, which increases the impact of CAP on patients and the health care system.

In our study, we found no relationship between the time of initiation of antibiotic therapy and mortality, possibly because of the long elapsed time to initiation of antibiotic therapy (a mean of $10 \mathrm{~h}$ ) and the low proportion of patients (19\%) who received antibiotic therapy within the first 4 hours. Difficulty in prompt initiation of antibiotic therapy had been reported in another study conducted in Brazil.(27) In the present study, we found that, even after the implementation of a protocol for the treatment of pneumonia, there was no success in the attempt to reduce the time to initiation of antibiotic therapy. This is probably due to a tendency to administer antibiotics at scheduled 
Table 1. Characteristics of the total study sample and subgroups. ${ }^{\text {a }}$

\begin{tabular}{|c|c|c|c|c|}
\hline \multirow[t]{2}{*}{ Variable } & \multirow{2}{*}{$\begin{array}{c}\text { Total } \\
(\mathbf{N}=304)\end{array}$} & \multicolumn{2}{|c|}{ In-hospital mortality } & \multirow[t]{2}{*}{ p } \\
\hline & & $\begin{array}{c}\text { Yes } \\
(n=47)\end{array}$ & $\begin{array}{c}\text { No } \\
(n=257)\end{array}$ & \\
\hline \multicolumn{5}{|l|}{ Gender } \\
\hline Male & $171(56.2)$ & $28(16.4)$ & $143(83.6)$ & 0.6 \\
\hline Female & $133(43.8)$ & $19(14.3)$ & $114(85.7)$ & \\
\hline Age, years & $67 \pm 17.3$ & $77.5 \pm 12.7$ & $65.2 \pm 17.3$ & 0.6 \\
\hline Race & & & & 0.7 \\
\hline White & $290(95.4)$ & $46(15.9)$ & $244(84.1)$ & \\
\hline Non-White & $14(4.6)$ & $1(7.1)$ & $13(92.9)$ & \\
\hline $\mathrm{CCl}$ score & $4.9 \pm 3.1$ & $8.11 \pm 2.8$ & $4.3 \pm 2.8$ & $<0.001$ \\
\hline CURB-65 score & $2.2 \pm 1.1$ & $3.0 \pm 1.0$ & $2.0 \pm 1.1$ & $<0.001$ \\
\hline PSI score & $107.2 \pm 50.6$ & $147.3 \pm 32.5$ & $99.9 \pm 50.0$ & $<0.001$ \\
\hline Smoking & $155(51.0)$ & $23(14.8)$ & $132(85.2)$ & 0.9 \\
\hline Dementia & $65(21.4)$ & $23(35.4)$ & $42(64.6)$ & $<0.001$ \\
\hline Diabetes & $46(15.5)$ & $8(17.4)$ & $132(85.2)$ & 0.7 \\
\hline Heart failure & $71(23.4)$ & $15(21.1)$ & $56(78.9)$ & 0.14 \\
\hline Neoplasia & $39(12.8)$ & $15(38.5)$ & $24(61.5)$ & $<0.001$ \\
\hline Renal disease & $34(11.2)$ & $13(38.2)$ & $21(61.8)$ & $<0.001$ \\
\hline Chronic lung disease & $150(49.3)$ & $23(15.3)$ & $127(84.7)$ & 1.0 \\
\hline Institutionalized & $22(7.2)$ & $7(31.8)$ & $15(68.2)$ & 0.03 \\
\hline
\end{tabular}

CCI: Charlson Comorbidity Index; CURB-65: mental Confusion, Urea, Respiratory rate, Blood pressure, and age $>$ 65 years; and PSI: Pneumonia Severity Index. ${ }^{a}$ Values expressed as $\mathrm{n}(\%)$ or as mean \pm SD.

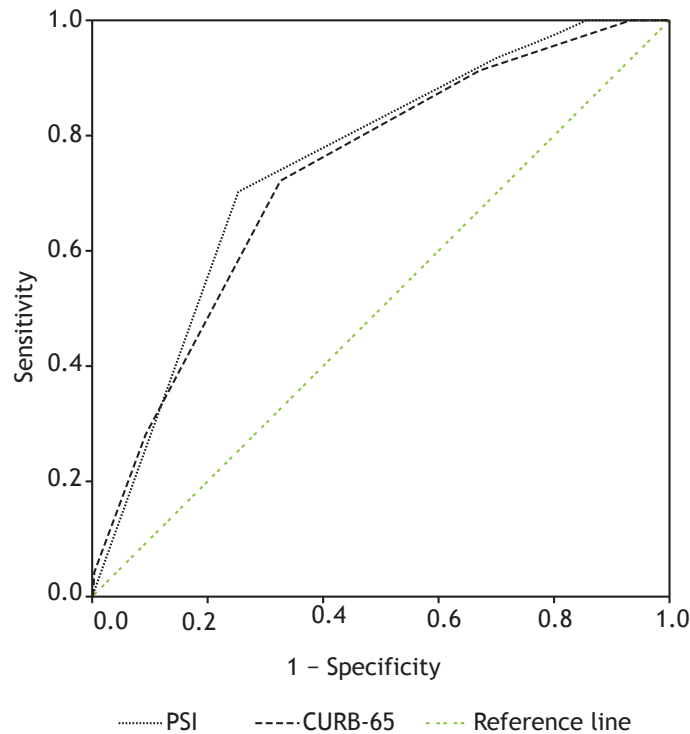

Figure 2. ROC curve of severity indices for prediction of in-hospital mortality. PSI: Pneumonia Severity Index; and CURB-65: mental Confusion, Urea, Respiratory rate, Blood pressure, and age $>65$ years.

times that are pre-determined by routine hospital protocols. Given that early initiation of medication is associated with better outcomes, with a study reporting a reduction of up to $30 \%$ in mortality when antibiotics are administered within the first hours of admission, ${ }^{(28)}$ efforts to reduce the time to treatment initiation are needed.

In a recent study, Rabello et al. ${ }^{(14)}$ reported that only $40 \%$ of the physicians used a validated prognostic score to assess patients with CAP, with clinical assessment alone remaining the most commonly used way to assess disease severity. In our study, the severity scores performed well in predicting in-hospital mortality, with areas under the ROC curve being 0.73 for the CURB65 score and 0.75 for the PSI, similarly to previous and recent international studies. ${ }^{(12,29)}$ These findings reinforce that these scores are useful for use in the Brazilian population as well and justify the efforts to promote their routine use in health care facilities.

The risk factors that remained associated with mortality after logistic regression were need for mechanical ventilation, a high CCI score, and a high CURB-65 score. The CCI is considered a good predictor of mortality in several settings, being an important marker of the patient's underlying health status, since it evaluates comorbidities in relation to acute infection. Although the CCI has been validated in several settings, including in patients with $\mathrm{COPD}_{1}{ }^{(30)}$ it has not commonly been reported as a risk factor for negative outcomes in other studies of CAP. The CURB-65 score, in addition to showing good prognostic ability, was independently associated with in-hospital mortality $(O R, 1.45)$. It is of note that the PSI did not show significance in the logistic regression model, probably because of the overlapping of variables assessed by the CURB-65 score and the CCI. Given that the CURB-65 score is much simpler (only five variables assessed) than the PSI (twenty variables assessed) and both are similar in terms of efficacy, the routine use of the CURB-65 score is justified. Lastly, need for mechanical ventilation is widely associated with worse outcomes, probably because it is an important marker of severity. 
Table 2. Factors associated with in-hospital mortality after multivariate analysis.

\begin{tabular}{lcc}
\multicolumn{1}{c}{ Factor } & OR $(95 \%$ CI) & p \\
Need for mechanical ventilation & $3.60(1.85-7.47)$ & $<0.001$ \\
CCI score & $1.30(1.18-1.43)$ & $<0.001$ \\
CURB-65 score & $1.46(1.09-1.98)$ & 0.006 \\
\hline
\end{tabular}

CCI: Charlson Comorbidity Index; and CURB-65: mental Confusion, Urea, Respiratory rate, Blood pressure, and age $>65$ years.

Our study has some limitations. The major limitation is that this was a single-center study involving a relatively small number of patients. However, our study is unique, since it is one of the few involving data from the interior of Brazil and involving all patients who were hospitalized for CAP over a one-year period, which is important in this disease with a seasonal variation. No patients were lost during the in-hospital follow-up period, and the severity profile of the patients was monitored in terms of underlying diseases, by using the CCI, and in terms of acute disease, by using the PSI and the CURB-65 score.
In conclusion, the findings of our study, such as the high in-hospital mortality rate and the need for ICU admission in almost on third of the patients, emphasize the impact that CAP has on individuals and the health care system. Individuals with a high burden of comorbidities, a high CURB-65 score, and a need for mechanical ventilation had a worse prognosis. Lastly, we observed delayed initiation of antibiotic therapy, even in a hospital setting. Measures to reduce the time to initiation of antibiotic therapy may result in better outcomes in this group of patients.

\section{REFERENCES}

1. Wunderink RG, Waterer GW. Community-acquired pneumonia. N Engl J Med. 2014.370(19):1863.

2. Brown SM, Jones BE, Jephson AR, Dean NC; Infectious Disease Society of America/American Thoracic Society 2007. Validation of the Infectious Disease Society of America/American Thoracic Society 2007 guidelines for severe community-acquired pneumonia. Crit Care Med, 2009.37(12):3010-6.

3. Akram AR, Chalmers JD, Hill AT. Predicting mortality with severity assessment tools in out-patients with community-acquired pneumonia. QJM. 2011;104(10):871-9. https://doi.org/10.1093/ qjmed/hcr088

4. Woodhead M, Welch CA, Harrison DA, Bellingan G, Ayres JG Community-acquired pneumonia on the intensive care unit: secondary analysis of 17,869 cases in the ICNARC Case Mix Programme Database. Crit Care. 2006;10 Suppl 2:S1. https://doi. org/10.1186/cc4927

5. Conde KA, Silva E, Silva CO, Ferreira E, Freitas FG, Castro I, et al Differences in sepsis treatment and outcomes between public and private hospitals in Brazil: a multicenter observational study PLoS One. 2013;8(6):e64790. https://doi.org/10.1371/journal. pone.0064790

6. Bozza FA, Salluh JI. An urban perspective on sepsis in developing countries. Lancet Infect Dis. 2010;10(5):290-1. https://doi. org/10.1016/S1473-3099(10)70074-8

7. Torres A, Blasi F, Dartois N, Akova M. Which individuals are at increased risk of pneumococcal disease and why? Impact of COPD, asthma, smoking, diabetes, and/or chronic heart disease on community-acquired pneumonia and invasive pneumococcal disease. Thorax. 2015;70(10):984-9. https://doi.org/10.1136/ thoraxjnl-2015-206780

8. Millett ER, De Stavola BL, Quint JK, Smeeth L, Thomas SL. Risk factors for hospital admission in the 28 days following a communityacquired pneumonia diagnosis in older adults, and their contribution to increasing hospitalisation rates over time: a cohort study. BMJ Open. 2015;5(12):e008737. https://doi.org/10.1136/bmjopen-2015-008737

9. Departamento de Informática do SUS - DATASUS [homepage on the Internet]. Brasília: Ministério da Saúde [cited 2017 Dec 1]. Morbidade Hospitalar do SUS - por local de residência - Brasil. Available from: http://tabnet.datasus.gov.br/cgi/tabcgi.exe?sih/cnv/niuf.def

10. Chalmers JD, Singanayagam A, Akram AR, Mandal P, Short PM, Choudhury G, et al. Severity assessment tools for predicting mortality in hospitalised patients with community-acquired pneumonia. Systematic review and meta-analysis. Thorax. 2010;65(10):878-83 https://doi.org/10.1136/thx.2009.133280

11. Lim WS, van der Eerden MM, Laing $R$, Boersma WG, Karalus $\mathrm{N}$, Town $\mathrm{Gl}$, et al. Defining community acquired pneumonia severity on presentation to hospital: an international derivation and validation study. Thorax. 2003;58(5):377-82. https://doi.org/10.1136/ thorax.58.5.377
12. Fine MJ, Auble TE, Yealy DM, Hanusa BH, Weissfeld LA, Singer DE, et al. A prediction rule to identify low-risk patients with communityacquired pneumonia. N Engl J Med. 1997;336(4):243-50. https://doi. org/10.1056/NEJM199701233360402

13. Ewig S, Ruiz M, Mensa J, Marcos MA, Martinez JA, Arancibia F, et al. Severe community-acquired pneumonia. Assessment of severity criteria. Am J Respir Crit Care Med. 1998;158(4):1102-8. https://doi. org/10.1164/ajrccm.158.4.9803114

14. Rabello L, Conceição C, Ebecken K, Lisboa T, Bozza FA, Soares $M$, et al., Management of severe community-acquired pneumonia in Brazil: a secondary analysis of an international survey. Rev Bras Ter Intensiva. 2015;27(1):57-63. https://doi.org/10.5935/0103 $507 X .20150010$

15. Menéndez R, Torres A, Zalacaín R, Aspa J, Martín Villasclaras JJ Borderías $L$, et al. Risk factors of treatment failure in community acquired pneumonia: implications for disease outcome. Thorax. 2004;59(11):960-5. https://doi.org/10.1136/thx.2003.017756

16. Marti C, Garin N, Grosgurin O, Poncet A, Combescure C, Carballo $\mathrm{S}$, et al. Prediction of severe community-acquired pneumonia: a systematic review and meta-analysis. Crit Care. 2012;16(4):R141. https://doi.org/10.1186/cc11447

17. Yandiola PPE, Capelastegui A, Quintana J, Diez R, Gorordo I, Bilbao $A$, et al. Prospective comparison of severity scores for predicting clinically relevant outcomes for patients hospitalized with community-acquired pneumonia. Chest. 2009;135(6):1572-1579. https://doi.org/10.1378/chest.08-2179

18. Donalisio MR, Arca $\mathrm{CH}$, Madureira PR. Clinical, epidemiological and etiological profile of inpatients with community-acquired pneumonia at a general hospital in the Sumaré microregion of Brazil. J Bras Pneumol. 2011;37(2):200-8. https://doi.org/10.1590/S180637132011000200010

19. Diogo LP, Bahlis LF, Wajner A, Waldemar FS. Decreased mortality in patients hospitalized due to respiratory diseases after installation of an intensive care unit in a secondary hospital in the interio of Brazil. Rev Bras Ter Intensiva. 2015;27(3):235-9. https://doi. org/10.5935/0103-507X.20150043

20. Mandell LA, Wunderink RG, Anzueto A, Bartlett JG, Campbell GD Dean NC, et al. Infectious Diseases Society of America/American Thoracic Society consensus guidelines on the management of community-acquired pneumonia in adults. Clin Infect Dis. 2007;44 Suppl 2:S27-72. https://doi.org/10.1086/511159

21. R Development Core Team. R: A language and environment for statistical computing. Vienna, Austria: R Foundation for Statistical Computing; 2017. Available from: http://www.R-project.org/

22. R Foundation for Statistical Computing [homepage on the Internet]. Vienna, Austria: R Foundation for Statistical Computing; [cited 2017 Dec 1]. Hmisc: Harrell Miscellaneous. Available from: https:// CRAN.R-project.org/package $=\mathrm{Hmisc}$ 
Clinical, epidemiological, and etiological profile of inpatients with community-acquired

pneumonia in a public hospital in the interior of Brazil

23. Robin X, Turck N, Hainard A, Tiberti N, Lisacek F, Sanchez JC, et al. pROC: an open-source package for $\mathrm{R}$ and $\mathrm{S}+$ to analyze and compare ROC curves. BMC Bioinformatics. 2011;12:77. https://doi. org/10.1186/1471-2105-12-77

24. Zeileis A. Econometric Computing with $\mathrm{HC}$ and HAC Covariance Matrix Estimators. J Statistic Software. 2004;11(10):1-17. https://doi. org/10.18637/jss.v011.i10

25. Silveira $C D$, Ferreira $C S$, Corrêa Rde A. Adherence to guidelines and its impact on outcomes in patients hospitalized with community-acquired pneumonia at a university hospital. J Bras Pneumol. 2012;38(2):148-57. https://doi.org/10.1590/S180637132012000200002

26. Dean NC, Bateman KA, Donnelly SM, Silver MP, Snow GL, Hale D. Improved clinical outcomes with utilization of a communityacquired pneumonia guideline. Chest. 2006;130(3):794-9. https://doi. org/10.1378/chest.130.3.794
27. Conterno LO, Moraes FY, Silva Filho CR. Implementation of community-acquired pneumonia guidelines at a public hospital in Brazil. J Bras Pneumol. 2011;37(2):152-9. https://doi.org/10.1590/ S1806-37132011000200004

28. Prina E, Ranzani OT, Torres A. Community-acquired pneumonia Lancet. 2015;386(9998):1097-108. https://doi.org/10.1016/S01406736(15)60733-4

29. Ito $A$, Ishida $T$, Tokumasu $H$, Washio $Y$, Yamazaki $A$, Ito $Y$, et al. Prognostic factors in hospitalized community-acquired pneumonia: a retrospective study of a prospective observational cohort. BMC Pulm Med. 2017;17(1):78. https://doi.org/10.1186/s12890-017-0424-4

30. Budweiser $S$, Harlacher M, Pfeifer M, Jörres RA. Co-morbidities and hyperinflation are independent risk factors of all-cause mortality in very severe COPD. COPD. 2014;11(4):388-400. https://doi.org/10.31 09/15412555.2013.836174 